\title{
Acute myocardial injury is common in patients with COVID-19 and impairs their prognosis
}

\author{
Jia-Fu Wei, ${ }^{1}$ Fang-Yang Huang (1) , ${ }^{1}$ Tian-Yuan Xiong, ${ }^{1}$ Qi Liu, ${ }^{1}$ Hong Chen, ${ }^{2}$ \\ Hui Wang, ${ }^{1}$ He Huang, ${ }^{1}$ Yi-Chun Luo, ${ }^{3}$ Xuan Zhou, ${ }^{3}$ Zhi-Yue Liu, ${ }^{1}$ Yong Peng, ${ }^{1}$ \\ Yuan-Ning $\mathrm{Xu}_{1}{ }^{1}$ Bo Wang, ${ }^{4}$ Ying-Ying Yang, ${ }^{5}$ Zong-An Liang, ${ }^{6}$ Xue-Zhong Lei, ${ }^{7}$ \\ Yang $\mathrm{Ge}^{8}$ Ming Yang, ${ }^{9}$ Ling Zhang, ${ }^{10}$ Ming-Quan Zeng, ${ }^{10} \mathrm{He} \mathrm{Yu}{ }^{7}$ Kai Liu, \\ Yu-Heng Jia, ${ }^{1}$ Bernard D Prendergast (1), ${ }^{11}$ Wei-Min Li, ${ }^{6}$ Mao Chen ${ }^{1}$
}

For numbered affiliations see end of article.

\section{Correspondence to Professor Mao Chen, Department of Cardiology, Sichuan University West China Hospital, Chengdu 610041, China: hmaochen@vip.sina.com Professor Wei-Min Li; weimi003@scu.edu.cn Professor Bernard D Prendergast: bernard.prendergast@gstt. nhs.uk \\ Professor He Huang; huanghe@wchscu.cn}

J-FW, F-YH, T-YX, QL, HC and HW contributed equally.

Received 30 March 2020 Revised 3 April 2020 Accepted 6 April 2020 Published Online First 30 April 2020

\section{Linked}

- http://dx.doi.org/10.1136/ heartjnl-2020-317025

\section{Check for updates}

(C) Author(s) (or their employer(s)) 2020. No commercial re-use. See rights and permissions. Published by BMJ.

To cite: Wei J-F, Huang F$Y$, Xiong T-Y, et al. Heart 2020;106:1154-1159.

\section{ABSTRACT}

Objective We sought to explore the prevalence and immediate clinical implications of acute myocardial injury in a cohort of patients with COVID-19 in a region of China where medical resources are less stressed than in Wuhan (the epicentre of the pandemic).

Methods We prospectively assessed the medical records, laboratory results, chest CT images and use of medication in a cohort of patients presenting to two designated covid-19 treatment centres in Sichuan, China. Outcomes of interest included death, admission to an intensive care unit (ICU), need for mechanical ventilation, treatment with vasoactive agents and classification of disease severity. Acute myocardial injury was defined by a value of high-sensitivity troponin T (hs-TnT) greater than the normal upper limit.

Results A total of 101 cases were enrolled from January to 10 March 2020 (average age 49 years, IQR 34-62 years). Acute myocardial injury was present in $15.8 \%$ of patients, nearly half of whom had a hs-TnT value fivefold greater than the normal upper limit. Patients with acute myocardial injury were older, with a higher prevalence of pre-existing cardiovascular disease and more likely to require ICU admission (62.5\% vs $24.7 \%, p=0.003)$, mechanical ventilation ( $43.5 \%$ vs $4.7 \%, p<0.001)$ and treatment with vasoactive agents (31.2\% vs 0\%, p<0.001). Log hs-TnT was associated with disease severity (OR 6.63, 95\% Cl 2.24 to 19.65), and all of the three deaths occurred in patients with acute myocardial injury.

Conclusion Acute myocardial injury is common in patients with COVID-19 and is associated with adverse prognosis.

\section{INTRODUCTION}

The recent outbreak of COVID-19 is a public health emergency of international concern and continues to spread worldwide. Knowledge concerning this threatening disease is accumulating rapidly, but many uncertainties remain, including detailed understanding of its extrapulmonary manifestations.

ACE2 has been identified as a functional receptor for coronaviruses and is highly expressed in the lungs as well as the heart. ${ }^{1}$ Electrocardiographic changes, troponin elevation and subclinical left ventricular diastolic impairment have been previously reported in patients with severe acute respiratory syndrome (SARS). ${ }^{2}{ }^{3}$ Similarly, acute myocardial injury (defined by elevated levels of cardiac biomarkers, troponin or creatine kinase (CK)) appears to be frequent during the current outbreak of COVID-19, ${ }^{4}$ although the mechanisms of injury and its clinical implications remain poorly defined. The majority of patients currently requiring hospital care for COVID-19 are categorised as nonsevere. ${ }^{5}$ It is therefore important to understand whether accompanying acute myocardial injury is a bystander phenomenon or an important contributor to immediate and long-term outcome with implications for clinical management.

Although recent reports have demonstrated the association of cardiac injury with mortality, these data arose from Wuhan, where medical resources have been most stressed, ${ }^{67}$ potentially augmenting the effect of cardiac injury due to delayed hospital admission and specialist management. Analysis of data from less stressed regions is also needed to confirm the broader significance of cardiac injury in COVID-19. Accordingly, we sought to characterise the prevalence and clinical implications of acute myocardial injury in a large cohort of patients with laboratory-confirmed COVID-19 in a region where clinical resources are under less pressure.

\section{METHODS}

\section{Study population}

We collected the data of 103 consecutive patients with laboratory-confirmed SARS-CoV-2 infection admitted to the Public Health Clinical Centre of Chengdu and West China Hospital, Sichuan University, between 16 January and 10 March 2020. Both institutions are certified COVID-19 tertiary care hospitals and designated hub centres within Sichuan province. Laboratory-confirmed cases were defined by a positive result on high-throughput sequencing or real-time reverse-transcriptase-PCR assay of nasal and/or pharyngeal swabs. ${ }^{8}$

\section{Data collection}

Medical records, use of medications and the findings of laboratory investigations, chest CT and transthoracic echocardiography (TTE), where available, were collected from all patients. Admission laboratory investigations included full blood 


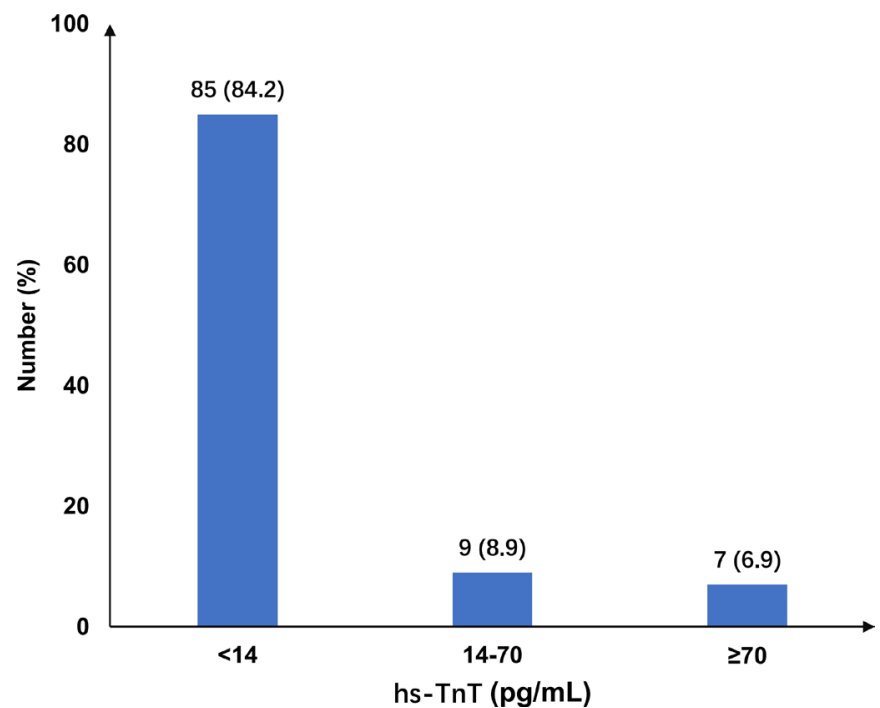

Figure 1 Distribution of hs-TnT values in the study cohort $(\mathrm{N}=101)$. hs-TnT, high-sensitivity toponin T.

count, biochemical analysis, coagulation testing, liver and renal function, inflammatory markers (C reactive protein (CRP) and procalcitonin (PCT)) and cardiac biomarkers (high-sensitivity troponin $\mathrm{T}$ (hs-TnT), creatine kinase and MB subfraction (CK$\mathrm{MB}$ ), N-terminal-proB-type natriuretic peptide (NT-proBNP) and lactate dehydrogenase (LDH)). TTE was performed if needed to guide clinical care, and all chest CT scans were evaluated by investigators blinded to patients' clinical status.

\section{Study outcomes}

Key outcomes included disease severity (see further), admission to an intensive care unit (ICU), need for mechanical ventilation or vasoactive agents, and death.

\section{Patient and public involvement}

Patients and the public were not involved in the study design, analysis, interpretation and writing of the study.

\section{Study definitions}

Severity of COVID-19 was classified at the time of admission using the novel coronavirus pneumonia diagnostic criteria and treatment regimens defined by the National Health Commission of the People's Republic of China, ${ }^{9}$ and reclassifed at the time of hospital discharge according to clinical evolution of the disease. These diagnostic criteria and treatment regimen have been recently updated to a seventh version, but criteria defining a severe/critical case remain unchanged from the fourth version (published before discharge of the first case in this cohort). Severe ( $\mathrm{r}$ espiratory rate, $\geq 30$ breaths/min; resting oxygen saturation, $\leq 93 \%$; or $\mathrm{PaO}_{2} / \mathrm{FiO}_{2}$ ratio, ie, oxygenation index, $\leq 300 \mathrm{~mm}$ $\mathrm{Hg}$ ) and critical (respiratory failure requiring mechanical ventilation, shock or need for intensive care) cases were grouped together for the purposes of this study. Acute myocardial injury was defined by an hs-TnT value greater than the institutional upper limit of normal $(14 \mathrm{pg} / \mathrm{mL})$. A single radiologist assessed the CT pulmonary lesion ratio (defined by the ratio of the sum of lesion areas on each slice to the total pulmonary tissue area) for all patients.

\section{Statistical analysis}

Continuous variables are expressed as medians (IQRs). Categorical variables are expressed as frequencies (percentages). Continuous variables were compared using the Student t-test or the Mann-Whitney U test, as appropriate, and categorical variables using $\chi^{2}$ test or the Fisher exact test, as appropriate. Since values of NT-proBNP and hs-TnT were not normally distributed, data were log-transformed for multivariate analyses. Pearson's correlation was used to test the association between ejection fraction and $\log$ hs-TnT. A multivariate linear regression analysis was carried out to explore independent factors associated with hs-TnT levels. Those with $\mathrm{p}<0.05$ in univariate analysis were introduced into the multivariate linear regression model. To investigate the impact of factors on disease severity, logistic regression analyses were carried out to determine ORs and 95\% CIs for covariates with severe/critical disease as the bivariate outcome. Considering the number of events, we did not further perform multivariate logistic regression. We considered age and gender, daily habit, comorbidities, use of medications, lab tests and pulmonary issues for candidate variables in both regression models, which were age and gender, smoking, use of ACE inhibitor (ACEi), angiotensin II receptor blocker (ARB) or calcium channel blocker (CCB), estimated glomerular filtration rate (eGFR), CRP, white blood cell count (WBC), log NT-proBNP, $\log$ hs-TnT, pulmonary lesion ratio, oxygenation index and pre-existing coronary artery disease, hypertension, diabetes mellitus or cerebrovascular disease. All statistical analyses were performed using STATA/MP V.16.0, and a two-sided $\mathrm{p}$ value of $<0.05$ was considered statistically significant.

\section{RESULTS}

Two patients were excluded from analysis due to missing data. Among 101 patients (average age 49 years, IQR 34-62 years) with complete datasets, $16(15.8 \%)$ had evidence of acute myocardial injury (hs-TnT $>14 \mathrm{pg} / \mathrm{mL}$ ) (figure 1). Baseline characteristics of patients with and without acute myocardial injury are displayed in table 1 . Patients with cardiac injury were older, with a higher prevalence of pre-existing cardiovascular disease (hypertension, coronary artery disease and cerebrovascular disease), presented with more significant dyspnoea and were more frequently treated with ACEi, ARB and CCB. They also had lower eGFR and oxygenation index, higher CRP, PCT, NT-proBNP and other cardiac biomarkers (CK, CK-MB and $\mathrm{LDH}$ ), and a greater pulmonary lesion ratio.

Univariate linear regression analyses using hs-TnT as a continuous variable demonstrated that age, use of ACEi or ARB, CRP, log NT-proBNP and pulmonary lesion ratio correlated positively with $\log$ hs-TnT (and inverse correlation with eGFR and oxygenation index) (table 2). In multivariate analysis, $\log$ hs-TnT was independently associated with the use of ACEi/ARB $(\beta=1.11, p=0.02)$ and eGFR $(\beta=-0.017$, $\mathrm{p}=0.006)$. Among patients with TTE data $(\mathrm{n}=32)$, there was a negative correlation between ejection fraction and log TnT-hs $(\mathrm{r}=-0.64, \mathrm{p}<0.001$; figure 2$)$.

Clinical outcomes are summarised in table 3 . Patients with acute myocardial injury were more frequently classified as severe/critical $(75.0 \%$ vs $29.4 \%, \mathrm{p}=0.001)$ and were more likely to require admission to ICU $(62.5 \%$ vs $24.7 \%, \mathrm{p}=0.003)$, mechanical ventilation $(43.5 \%$ vs $4.7 \%, \mathrm{p}<0.001)$ and vasoactive agents $(31.2 \%$ vs $0 \%, \mathrm{p}<0.001)$. Furthermore, all of the three deaths occurred in patients with acute myocardial injury. Older age, hypertension, cerebrovascular disease, the use of CCB, lower eGFR, and elevated NT-proBNP, hs-TnT 
Table 1 Baseline characteristics of patients with and without acute myocardial injury

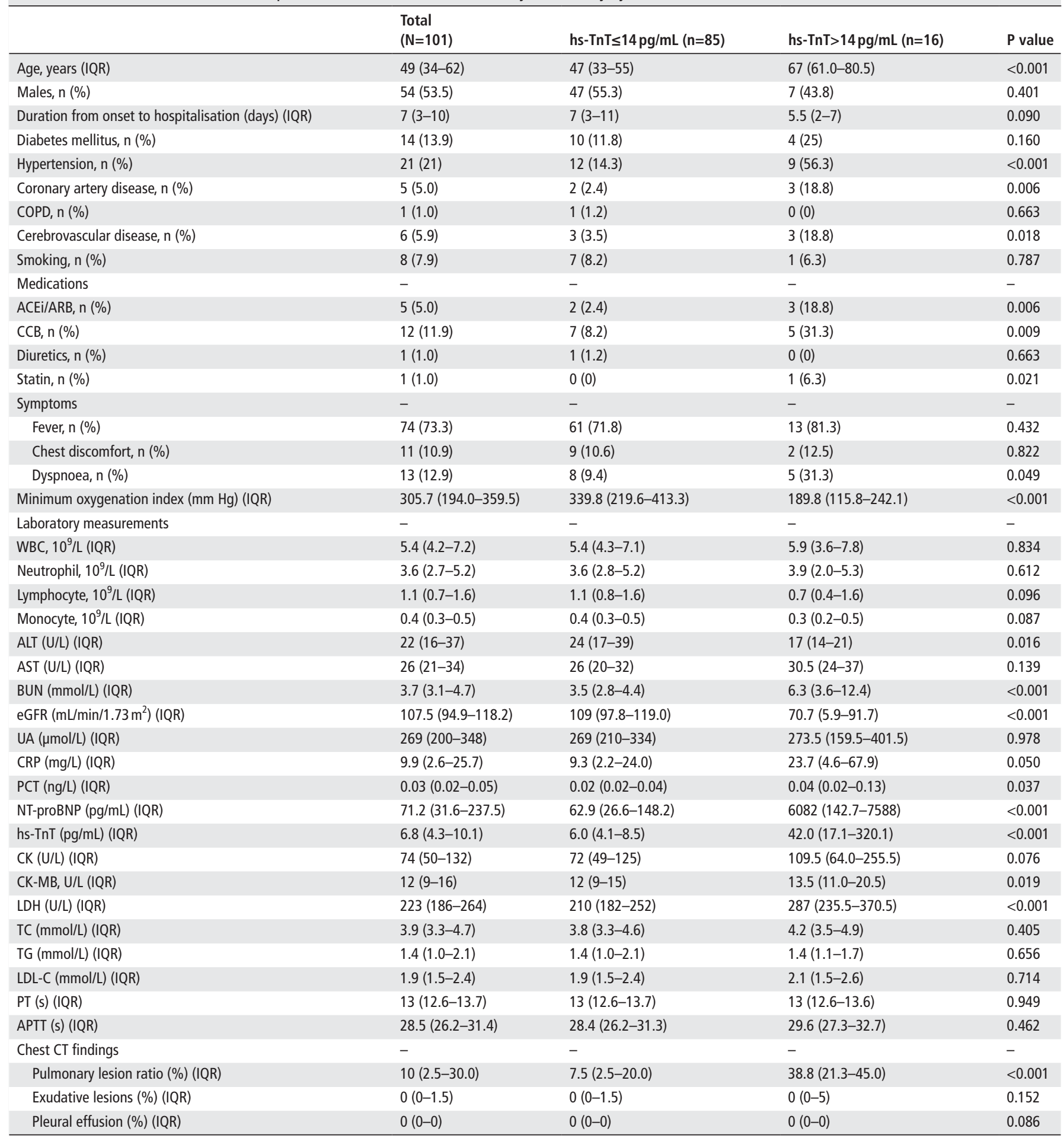

ACEi, ACE inhibitor; ALT, alanine aminotransferase; APTT, activated partial thromboplastin time; ARB, angiotensin II receptor blocker; AST, aspartate aminotransferase; BUN, blood urea nitrogen; $C C B$, calcium channel blocker; $C K$, creatine kinase; $C K-M B$, creatine kinase and MB subfraction; COPD, chronic obstructive pulmonary disease; CRP, C reactive protein; eGFR, estimated glomerular filtration rate; hs-TnT, high-sensitivity troponin T; LDH, lactate dehydrogenase; LDL-C, low-density lipoprotein cholesterol; NT-proBNP, Nterminal-proB-type natriuretic peptide; PCT, procalcitonin; PT, prothrombin time; TC, total cholesterol; TG, total triglyceride; UA, uric acid; WBC, white blood cell count.

and CRP were predictors of severe disease in the univariate analysis (table 4). All cases with the history of coronary artery disease were classified as severe cases.

\section{DISCUSSION}

Our study provides structured information concerning the role of acute myocardial injury in the setting of COVID-19 in regions affected by the pandemic but where resources are less stressed. We demonstrate that elderly patients with pre-existing cardiovascular disease and using antihypertensive drugs (including ACEi and ARB) are more likely to present with acute myocardial injury, although with a lower incidence than those in Wuhan, the epicentre of the pandemic (15.8\% vs $19.7 \%-27.8 \%)$, which in turn leads to more severe disease manifestations, including increased need for 


\begin{tabular}{|c|c|c|c|c|}
\hline \multirow[b]{2}{*}{ Log hs-TnT } & \multicolumn{2}{|c|}{ Univariate analysis } & \multicolumn{2}{|c|}{ Multivariate analysis } \\
\hline & B (SE) & $P$ value & B (SE) & $P$ value \\
\hline Age & $0.030(0.006)$ & 0.000 & $-0.007(0.008)$ & 0.430 \\
\hline Males & $-0.090(0.260)$ & 0.730 & & \\
\hline Smoking & $0.116(0.446)$ & 0.794 & & \\
\hline Use of ACEi/ARB & $2.584(0.479)$ & 0.000 & $1.110(0.468)$ & 0.020 \\
\hline Use of CCB & $1.066(0.370)$ & 0.005 & $-0.782(0.429)$ & 0.072 \\
\hline WBC & $-0.001(0.046)$ & 0.976 & & \\
\hline eGFR & $-0.018(0.003)$ & 0.000 & $-0.017(0.006)$ & 0.006 \\
\hline CRP & $0.018(0.004)$ & 0.000 & $0.002(0.004)$ & 0.593 \\
\hline Pulmonary lesion ratio & $0.036(0.006)$ & 0.000 & $0.013(0.007)$ & 0.072 \\
\hline Oxygenation index & $-0.005(0.001)$ & 0.000 & $-0.001(0.001)$ & 0.356 \\
\hline Diabetes mellitus & $0.561(0.345)$ & 0.108 & & \\
\hline Coronary artery disease & $1.730(0.521)$ & 0.001 & $0.573(0.457)$ & 0.214 \\
\hline Hypertension & $1.444(0.265)$ & $<0.001$ & $0.589(0.356)$ & 0.103 \\
\hline Cerebrovascular disease & $1.199(0.492)$ & 0.017 & $0.642(0.420)$ & 0.130 \\
\hline
\end{tabular}

ACEi, ACE inhibitor; ARB, angiotensin II receptor blocker; CCB, calcium channel blocker;

CRP, $C$ reactive protein; eGFR, estimated glomerular filtration rate; hs-TnT, high-sensitivity troponin T; WBC, white blood cell count.

mechanical ventilation and vasoactive agents, and a higher risk of death.

Within the past two decades, coronaviruses and influenza viruses have hit the world several times, causing significant mortality, economic loss and global panic. The true health burden may be underestimated since extrapulmonary manifestations are frequent. Acute cardiovascular complications of pneumonia are common and result from various mechanisms, including relative ischaemia, systemic inflammation and pathogen-mediated damage. ${ }^{10}$ Troponin is a widely accepted biomarker of myocardial injury, and elevated serum levels have been a notable feature during recent epidemics of respiratory virus infections. ${ }^{11-13}$ Similarly, acute myocardial injury has been demonstrated in $7.2 \%-12 \%$ of patients with COVID-19 in preliminary reports, ${ }^{8} 14$ with a higher prevalence among those requiring intensive care. Troponin elevation was transient in some studies $^{13}$ and associated with reversible subclinical left ventricular diastolic impairment in SARS, ${ }^{3}$ suggesting that it may be an innocent bystander phenomenon. However, patients with COVID-19 frequently present with initial cardiovascular symptoms, such as palpitations and chest tightness, ${ }^{4}$ and fulminant myocarditis has been reported as a cause of death, ${ }^{15}$ suggesting a complex interaction between this respiratory virus and the cardiovascular system. A study clearly delineating the clinical implications of troponin elevation in COVID-19 is therefore of considerable value.

COVID-19 is principally a lung pathogen and the pulmonary functional status is the principal determinant of classification of disease severity, clinical course and prognosis. ${ }^{16}$ Although the proportion of severe cases was around $15 \%$ in a recent nationwide study, ${ }^{1}$ major adverse outcomes (including ICU admission

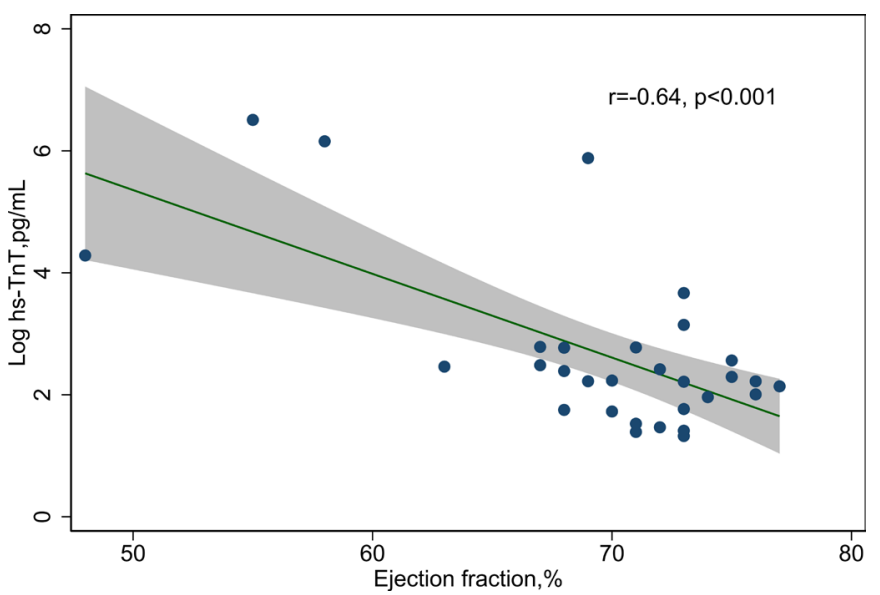

Figure 2 Correlation between ejection fraction and $\log \mathrm{hs}-\operatorname{TnT}(\mathrm{n}=32)$. hs-TnT, high-sensitivity toponin T.

and death) were also frequent in non-severe cases. Given that the majority of cases are non-severe, clear management strategies for this subgroup of patients are of vital importance, particularly as COVID-19 spreads worldwide. Our analysis demonstrates that acute myocardial injury complicating COVID-19 infection (indicated by troponin elevation) is associated with a considerable disease burden, including classification as a severe/critical case, increased need for mechanical ventilation and use of vasoactive agents, and a higher risk of death.

Cardiac injury in itself may reflect an ongoing pathological insult due to cytokine release or secondary hypoxaemia. ${ }^{4}$ Conversely, a florid systemic inflammatory response or immune paresis in patients with cardiac injury is likely to be associated with more severe pulmonary manifestations. This hypothesis is partly supported by our observation that patients with acute myocardial injury also demonstrated higher levels of CRP and PCT and a greater pulmonary lesion ratio.

Patients with acute myocardial injury were generally the elderly, with a higher likelihood of pre-existent cardiovascular comorbidities and use of antihypertensive drugs, such as ACEi and ARB. Age and comorbidities are consistently identified as risk factors for adverse events in outbreaks of respiratory virus infection. Indeed, the severity of the primary respiratory syndrome and risk of adverse outcomes in Middle East respiratory syndrome was increased in patients with pre-existing cardiovascular disease. ${ }^{17}$ Mortality data from 44672 cases of COVID-19 released by the Chinese Centre for Disease Control and Prevention demonstrate that patients with cardiovascular comorbidities have a much higher mortality $(10.5 \%$ vs $0.9 \%) .{ }^{18}$ The background clinical profile of patients with acute myocardial injury may also facilitate direct virus-mediated cardiotoxicity or secondary myocardial involvement in a systemic inflammatory response. Increased prevalence of pre-existing

Table 3 In-hospital clinical outcomes

\begin{tabular}{|c|c|c|c|c|}
\hline & $\begin{array}{l}\text { Total } \\
(\mathrm{N}=101)\end{array}$ & hs-TnT $\leq 14 \mathrm{pg} / \mathrm{mL}(\mathrm{n}=85)$ & hs-TnT>14 pg/mL $(n=16)$ & $P$ value \\
\hline Death, $n(\%)$ & $3(3.0)$ & 0 & $3(18.8)$ & $<0.001$ \\
\hline Severe case, $\mathrm{n}(\%)$ & $37(36.6)$ & $25(29.4)$ & $12(75)$ & 0.001 \\
\hline Admission to ICU, $\mathrm{n}(\%)$ & $31(30.7)$ & $21(24.7)$ & $10(62.5)$ & 0.003 \\
\hline Mechanical ventilation, $\mathrm{n}(\%)$ & $11(10.9)$ & $4(4.7)$ & $7(43.5)$ & $<0.001$ \\
\hline Vasoactive agents, $\mathrm{n}(\%)$ & $5(5.0)$ & 0 & $5(31.2)$ & $<0.001$ \\
\hline ICU duration, mean (SD) & $3.26(6.16)$ & $2.86(5.56)$ & $5.38(8.61)$ & 0.135 \\
\hline
\end{tabular}

$\mathrm{ICU}$, intensive care unit. 


\begin{tabular}{|c|c|c|}
\hline & \multicolumn{2}{|l|}{ Univariate } \\
\hline & OR $(95 \% \mathrm{Cl})$ & $P$ value \\
\hline Age & 1.07 (1.04 to 1.1$)$ & $<0.001$ \\
\hline Males & 1.47 (0.3 to 1.54$)$ & 0.357 \\
\hline Hypertension & 5.36 (1.91 to 15.04$)$ & 0.001 \\
\hline Diabetes mellitus & 2.76 (0.88 to 8.69$)$ & 0.083 \\
\hline Cerebrovascular disease & $10.16(1.14$ to 90.6$)$ & 0.038 \\
\hline Smoking & $3.28(0.74$ to 14.61$)$ & 0.119 \\
\hline Use of ACEi/ARB & 2.82 (0.45 to 17.72$)$ & 0.268 \\
\hline Use of CCB & 4.28 (1.19 to 15.36$)$ & 0.026 \\
\hline WBC & 1.07 (0.93 to 1.24$)$ & 0.334 \\
\hline CRP & 1.02 (1.01 to 1.04$)$ & 0.003 \\
\hline eGFR & 0.97 (0.95 to 0.99$)$ & 0.001 \\
\hline Log NT-proBNP & 2.80 (1.74 to 4.52$)$ & $<0.001$ \\
\hline Log hs-TnT & 6.63 (2.24 to 19.65$)$ & 0.001 \\
\hline Pulmonary lesion ratio & 1.10 (1.07 to 1.15$)$ & $<0.001$ \\
\hline
\end{tabular}

ACEi, ACE inhibitor; ARB, angiotensin II receptor blocker; $C C B$, calcium channel blocker; CRP, C reactive protein; eGFR, estimated glomerular filtration rate; $\mathrm{hs}$ - TnT, high-sensitivity troponin T; NT-proBNP, N-terminal-proB-type natriuretic peptide; WBC, white blood cell count.

cardiovascular disease is also likely to explain the more frequent use of ACEi/ARB in the group of patients with acute myocardial injury. ACE2 is the functional receptor for SARS-CoV-2, and increased levels associated with the use of renin-angiotensin-aldosterone system inhibitors ${ }^{19}$ might increase the possibility of virusmediated myocardial damage. However, this explanation remains hypothetical, and there are no current reliable data to justify alteration of ACEi/ARB treatment in patients with COVID-19.

Hubei Province (Wuhan) has an over 100-fold greater number of COVID-19 cases than Sichuan Province. The surge of cases needing medical care, especially in the early period of this outbreak, posed a major challenge to the regional healthcare system. Our results further support the association of cardiac injury with adverse events in patients with covid-19. ${ }^{6720}$ Furthermore, the disparity in the number of cases between the adjacent regions of Hubei and Sichuan Provinces clearly demonstrates the public health advantages of 'lockdown' in the national and international management of the COVID-19 outbreak.

Our study has inevitable limitations at this early stage of the COVID-19 outbreak. With a small sample size, we are unable to draw definitive conclusions and acknowledge the existence of selection bias during this very early study period in specialist hub centres. Owing to circumstances, complete documentation of exposure history and laboratory testing was not available for every patient, and echocardiography was only performed for clinical expediency. As a consequence, we were only able to define acute myocardial injury by troponin elevation without detailing myocardial tissue characteristics and haemodynamic function. Caution should be taken when interpreting the multivariable analysis of hs-TnT since the model is likely underpowered to assess independent associations for other predictors in the model.

\section{CONCLUSIONS}

Acute myocardial injury, as indicated by troponin elevation, is common in patients with COVID-19, particularly in the elderly with pre-existing cardiovascular comorbidities, and is associated with increased risk of progressive severe disease, need for ICU admission, mechanical ventilation and use of vasoactive agents, and death. Targeted treatment and preventative strategies are urgently needed for this vulnerable patient group.
Key messages

What is already known on this subject?

- The involvement of the cardiovascular system in patients with fatal coronavirus infections have been reported during the outbreaks of severe acute respiratory syndrome and Middle East respiratory syndrome. During COVID-19, the incidence of acute cardiac injury, as indicated by the elevation of troponin, ranged from $7.2 \%$ to $27.8 \%$. In cohorts from Wuhan (the epicentre of the pandemic), the association of cardiac injury with mortality has been reported.

\section{What might this study add?}

- This study was conducted in regions where medical resources were less stressed than Wuhan. In a cohort of 101 laboratoryconfirmed COVID-19 cases, acute myocardial injury (highsensitivity troponin $\mathrm{T}$ (hs-TnT) $>14 \mathrm{pg} / \mathrm{mL}$ ) was present in $15.8 \%$ of patients. Patients with acute myocardial injury were older, with a higher prevalence of pre-existing cardiovascular disease, and were more likely to require intensive care unit (ICU) admission ( $62.5 \%$ vs $24.7 \%, \mathrm{p}=0.003)$, mechanical ventilation $(43.5 \%$ vs $4.7 \%, p<0.001)$ and treatment with vasoactive agents $(31.2 \%$ vs $0 \%, p<0.001)$. Log hs-TnT was associated with disease severity (OR $6.63,95 \% \mathrm{Cl} 2.24$ to 19.65), and all of the three deaths in our cohort occurred in patients with acute myocardial injury.

\section{How might this impact on clinical practice?}

- Acute myocardial injury is common in patients with COVID-19, particularly in the elderly with pre-existing cardiovascular comorbidities, and is associated with increased risk of progressive severe disease, need for ICU admission, mechanical ventilation and use of vasoactive agents, and death. This subgroup of cases may need targeted treatment and preventative strategies.

\section{Author affiliations}

'Department of Cardiology, West China Hospital, Sichuan University, Chengdu, China

${ }^{2}$ The Public Health Clinical Center of Chengdu, Chengdu, Sichuan Province, China ${ }^{3}$ Department of Radiology, West China Hospital, Sichuan University, Chengdu, China ${ }^{4}$ Intensive Care Unit, West China Hospital, Sichuan University, Chengdu, China ${ }^{5}$ Department of Nephrology, West China Hospital, Sichuan University, Chengdu, China

${ }^{6}$ Department of Respiratory and Critical Care Medicine, West China Hospital, Sichuan University, Chengdu, China

${ }^{7}$ Center of Infectious Disease, West China Hospital, Sichuan University, Chengdu, China

${ }^{8}$ Department of Functional Examination Room, The Public Health Clinical Center of Chengdu, Chengdu, Sichuan Province, China

${ }^{9}$ Department of Respiratory Medicine, The Public Health Clinical Center of Chengdu, Chengdu, Sichuan Province, China

${ }^{10}$ Intensive Care Unit, The Public Health Clinical Center of Chengdu, Chengdu, Sichuan Province, China

'Department of Cardiology, St Thomas' Hospital, London, UK

Acknowledgements We thank the covid-19 Clinical Taskforce, Sichuan Province, and all the health care providers and institutions involved in the management of patients with covid-19.

Contributors Conception and design: MC, BDP, W-ML and HH. Data acquisition: YP, Y-NX, HC, Y-YY, BW, YG, M-QZ, MY, HY, KL, LZ and Y-HJ. Imaging process and analysis: $L Z, Y-C L$ and $X Z$. Analysis of echocardiography: HW, HH and Z-YL. Analysis and interpretation of the data: J-FW, F-YH, QL and Y-CL. Drafting of the manuscript: J-FW, F-YH and T-YX. Critical revision for important intellectual content and final approval of the manuscript: all authors. Responsible for the overall content as the guarantors: MC and WML. 
Funding The authors have not declared a specific grant for this research from any funding agency in the public, commercial or not-for-profit sectors.

Competing interests None declared.

Patient and public involvement Patients and/or the public were not involved in the design, or conduct, or reporting, or dissemination plans of this research.

Patient consent for publication Not required.

Ethics approval The study was approved by the institutional ethics committee (study number 2020-226) and all patients provided written informed consent

Provenance and peer review Not commissioned; internally peer reviewed.

Data availability statement Data are available upon reasonable request. The datasets used during the current study are available from the corresponding author on reasonable request. Individual participant data without names and identifiers that underlie the result reported in this article are available on request. The data will be available for others to request immediately after publication of the article. The proposal with detailed description of study objectives will be needed for evaluation of the reasonability to request our data. The data requestors will need to sign a data access agreement to gain the datasets. The proposals should be directed to hmaochen@vip.sina.com.

This article is made freely available for use in accordance with BMJ's website terms and conditions for the duration of the covid-19 pandemic or until otherwise determined by BMJ. You may use, download and print the article for any lawful, non-commercial purpose (including text and data mining) provided that all copyright notices and trade marks are retained.

ORCID iDs

Fang-Yang Huang http://orcid.org/0000-0002-3049-2996

Bernard D Prendergast http://orcid.org/0000-0002-6031-2124

\section{REFERENCES}

1 Turner AJ, Hiscox JA, Hooper NM. Ace2: from vasopeptidase to SARS virus receptor. Trends Pharmacol Sci 2004;25:291-4.

2 Yu C-M, Wong RS-M, Wu EB, et al. Cardiovascular complications of severe acute respiratory syndrome. Postgrad Med J 2006;82:140-4.

3 Li SS-lung, Cheng C-wah, Fu C-lai, et al. Left ventricular performance in patients with severe acute respiratory syndrome: a 30-day echocardiographic follow-up study. Circulation 2003:108:1798-803.

4 Zheng Y-Y, Ma Y-T, Zhang J-Y, et al. COVID-19 and the cardiovascular system. Nat Rev Cardiol;395.
5 Guan W-jie, Ni Z-yi, Hu Y, et al. Clinical characteristics of coronavirus disease 2019 in China. N Engl I Med Overseas Ed.

6 Shi S, Qin M, Shen B, et al. Association of cardiac injury with mortality in hospitalized patients with COVID-19 in Wuhan, China. JAMA Cardiol.

7 Guo T, Fan Y, Chen M, et al. Cardiovascular implications of fatal outcomes of patients with coronavirus disease 2019 (COVID-19). JAMA Cardiol 2020. doi:10.1001/ jamacardio.2020.1017. [Epub ahead of print: 27 Mar 2020].

8 Huang C, Wang Y, Li X, et al. Clinical features of patients infected with 2019 novel coronavirus in Wuhan, China. The Lancet 2020;395:497-506.

9 National Health Commission of the People's Republic of China. Novel CoronavirusPneumonia diagnostic and treatment regimens. Available: http://www.nhc.gov.cn/ yzygj/s7653p/new_list.shtml

10 Restrepo MI, Reyes LF. Pneumonia as a cardiovascular disease: Pneumonia-related cardiac damage. Respirology 2018;23:250-9.

11 Guan Y-juan, Tang X-ping, Yin C-biao, et al. [Study on the myocardiac injury in patients with severe acute respiratory syndrome]. Zhonghua Nei Ke Za Zhi 2003:42:458-60.

12 Alhogbani T. Acute myocarditis associated with novel middle East respiratory syndrome coronavirus. Ann Saudi Med 2016:36:78-80.

13 Han J, Mou Y, Yan D, et al. Transient cardiac injury during H7N9 infection. Eur J Clin Invest 2015:45:117-25.

14 Wang D, Hu B, Hu C, et al. Clinical characteristics of 138 hospitalized patients with 2019 novel coronavirus-infected pneumonia in Wuhan, China. JAMA 2020;07:1061-9.

15 Ruan Q, Yang K, Wang W, et al. Clinical predictors of mortality due to COVID-19 based on an analysis of data of 150 patients from Wuhan, China. Intensive Care Med.

16 Metlay JP, Waterer GW, Long AC, et al. Diagnosis and treatment of adults with community-acquired pneumonia. An official clinical practice guideline of the American thoracic Society and infectious diseases Society of America. Am J Respir Crit Care Med 2019:200:e45-67.

17 Badawi A, Ryoo SG. Prevalence of comorbidities in the middle East respiratory syndrome coronavirus (MERS-CoV): a systematic review and meta-analysis. Int I Infect Dis 2016;49:129-33

18 Novel Coronavirus Pneumonia Emergency Response Epidemiology Team. [The epidemiological characteristics of an outbreak of 2019 novel coronavirus diseases (COVID-19) in Chinal. Zhonghua Liu Xing Bing Xue Za Zhi 2020:41:145-51.

19 Ferrario CM, Jessup J, Chappell MC, et al. Effect of angiotensin-converting enzyme inhibition and angiotensin II receptor blockers on cardiac angiotensin-converting enzyme 2. Circulation 2005;111:2605-10.

20 Lippi G, Lavie CJ, Sanchis-Gomar F. Cardiac troponin I in patients with coronavirus disease 2019 (COVID-19): evidence from a meta-analysis. Prog Cardiovasc Dis 2020. doi:10.1016/j.pcad.2020.03.001. [Epub ahead of print: 10 Mar 2020]. 